\title{
BUILD FOR ALL
}

\author{
by
}

Katlynn Sverko

BA, Creative Industries, Ryerson University, 2017

\author{
An MRP \\ presented to Ryerson University \\ in partial fulfillment of the \\ requirements for the degree of \\ Master of Digital Media \\ in the program of \\ Digital Media
}

Toronto, Ontario, Canada, 2018

(C) Katlynn Sverko, 2018 


\section{AUTHOR'S DECLARATION FOR ELECTRONIC SUBMISSION OF AN MRP}

I hereby declare that I am the sole author of this MRP. This is a true copy of the MRP, including any required final revisions.

I authorize Ryerson University to lend this MRP to other institutions or individuals for the purpose of scholarly research.

I further authorize Ryerson University to reproduce this MRP by photocopying or by other

means, in total or in part, at the request of other institutions or individuals for the purpose of scholarly research.

I understand that my MRP may be made electronically available to the public. 


\title{
BUILD FOR ALL
}

\author{
Katlynn Sverko \\ Master of Digital Media \\ Digital Media \\ Ryerson University, 2018
}

\begin{abstract}
Internet access is being seen more and more as a basic human right. Yet the Internet remains inaccessible for many. Guidelines on accessibility exist, as do penalties for noncompliance, yet $84 \%$ of startup websites remain inaccessible. Accessibility is the capacity for individuals to equivalently use goods and services. The purpose of this research is to determine if exposure to an online resource focusing on how to make startups accessible, rather than why, impacts the willingness of individuals to adopt accessible strategies. Through the use of pre- and postsurveys, the impact on willingness was measured and compared to pre-exposure levels. The results suggest that startups may be willing to adopt accessibility guidelines after explicitly being shown how, however a gap still remains between willingness and execution.
\end{abstract}




\section{Acknowledgements}

Thank you to my supervisor, Dr. Sean Wise, for your expert guidance, support, and for all the time you spent to make sure this MRP was headed in the right direction. Thank you to Ahmed Sagarwala for your continued support and direction. I would also like to thank Dr. Michael Carter for encouraging me to pursue MDM and taking the time to straighten my priorities. Thank you to Ryerson's Chang School and the Accessibility Project team, namely Dr. Elaine Lam and Darren Cooper, for being the fuel I needed to get Build for All off the ground. Thank you to Harrison Dearden for being a great source of perspective and for being my second reader. Finally, I would like to thank my fiancé Samuel Legros, without you Build for All would not exist. Thank you all. 


\section{TABLE OF CONTENTS}

$\begin{array}{lll}\text { ABSTRACT } & \text { iii }\end{array}$

ACKNOWLEDGEMENTS

LIST OF APPENDICES Vi

1. INTRODUCTION 1

2. REVIEW OF LITERATURE 6

3. METHODOLOGY 9

4. FINDINGS 11

5. ANALYSIS 13

6. DISCUSSION 15

7. CONCLUSION 17

$\begin{array}{lr}\text { APPENDICES } & 19\end{array}$

$\begin{array}{ll}\text { REFERENCES } & 24\end{array}$ 


\section{List of Appendices}

Appendix A Startup Websites that Failed Accessibility Standards 19

Appendix B Average Number of Errors Startup Websites 20

Appendix C Build for All Screenshot: Developing Accessible Teams 21

Appendix D Build for All Screenshot: How-To Instructions 22

Appendix E Build for All Screenshot: Job Specific Categories 23 


\section{Introduction}

According to Miniwatts Marketing Group (2018), there are over 4 billion people

accessing the Internet. Pilling, Barrett, and Floyd (2004) report that up to 7\% of the population in the United States, aged 25 and over, face accessibility challenges in relation to Internet-usage. The United Nations Human Rights Council (2011) declares access to the internet a human right, and charges government bodies, companies, and individuals with making the "Internet widely available, accessible and affordable to all” (para. 66). According to the Disability Rights Commission (2004) 81\% of websites do not meet even the most basic Web Accessibility Initiative (WAI) requirements. 92\% of government organizations in the United States are currently not accessible (Information Technology and Innovation Foundation, 2017). In 2017, there were 814 federal lawsuits for claims against inaccessible websites in the United States alone (Vu \& Ryan, 2018). With less than $20 \%$ of websites meeting the minimum requirements for web accessibility, one must ask why are not all websites accessible? How can the accessibility and inclusivity of websites increase? Build for All's purpose is to increase inclusivity and accessibility in online products and services created by startups, by acting as a resource for startups to include accessibility practices.

Build for All is an online resource for making startup websites more accessible. It was specifically created for the purposes of this research. This is done by providing articles on how to make websites accessible with explicit examples targeted at chief executive officers, developers, communication officers, chief technology officers, and marketers within a startup as they have the power to adopt or initiate accessible practices. Build for All also provides tools to facilitate the education and practice adoption, tools include: web auditing tools, shortcuts to policies and regulations, and accessibility definitions. 


\section{Background}

\section{The Current State:}

First and foremost, it must be stated that resources pertaining to accessibility do exist. The WAI began as project created by the World Wide Web Consortium (W3C) in 1997 in order to create a more accessible internet (Dardailler, 2009). In order to create and contribute to the discussion of web accessibility as it pertains to policy, W3C created the Web Content Accessibility Guidelines (WCAG) which is currently in its second iteration known as WCAG 2.0 (2008). WCAG 2.0 Level AA becomes the minimum operating standard by the AODA (2014) in Ontario by 2021. This was the genesis of this project. Build for All is a fully accessible online platform that provides resources for those interested in creating accessible projects and companies. Resources on Build for All are specifically targeted at startups and those in the roles of developer, designer, marketer, or CEO.

The WAI (2016) describes accessibility as addressing "discriminatory aspects related to equivalent user experience for people with disabilities, including people with age-related impairments". In Ontario, Canada there is a law requiring businesses to be accessible called the Accessibility for Ontarians with Disabilities Act (AODA), an act that aims to make Ontario fully accessible, both online and in physical locations by 2025 (2014, para. 1a). For the web, accessibility means that persons with disabilities can "perceive, understand, navigate, and interact with websites and tools, and that they can contribute equally without barriers" (WAI, 2016). User experience, frequently referred to as usability, is the ability for the user to reach their objective effectively through intelligent design (WAI, 2016). The terms inclusion, inclusivity, and universal design address that humans have differing abilities, and that products and services have the ability to include everyone through intelligent and considerate design (WAI, 2016; Norman, 2013). In relation to the web and the purposes of this research, the term disability refers 
to any condition, both long term and short term, that disenfranchises an individual's ability, whether mentally or physically, to use the web in any way. In order to discover the gap between these resources and implementation, through initial discovery interviews I looked at how each of the pre-existing solutions listed above addressed increasing accessibility, user awareness, and considered if these resources were successful in their methods. Success was determined by whether the startup implemented accessibility using the resource, though there was not enough time in order to follow-up with adoption so I focused on willingness to adopt accessibility practices.

\section{The Initial Interviews:}

Establishing interviews with 20 developers, 3 CEOs, and 8 designers from various startups showed that startups considered physical accessibility to their office space, however startups showed little consideration for the accessibility of their websites. Through analyzing the content and accessibility of 63 websites of startups based out of the DMZ at Ryerson University, a university based technology incubator in Toronto, Ontario, only $16 \%$ received a passing grade on accessibility based on the WCAG 2.0 Level AA standard (see Appendix A). The average number of errors on these websites was 27 (See Appendix B). From the interviews, the most common barriers included not knowing which resource to follow, and not knowing how to go about making their websites accessible. Build for All is a response to these barriers. Explicit instructions and examples were created for Build for All in order to increase comprehension of how to make startup websites accessible. Explicit instructions take "relevant internal and external knowledge, dissemination, and editing/processing to make it more usable" (Spencer, 1997, p. 2).

With the available accessibility documentation and options there are no online resources available that target the iteration of accessible and inclusive development of startups and 
businesses, with actionable steps and examples. $0 \%$ of resources focused on accessibility for startups. The WAI, WCAG 2.0, and AODA, are focused on $\boldsymbol{w h} \boldsymbol{y}$ websites should be made accessible, and not on how. In response to this the Build for All online platform was created. Build for All contains information regarding the development of accessible teams, products, and environments (See Appendix C). Actionable content, such as instructions and examples, have been created for Build for All regarding accessibility, as it pertains to the web accessibility requirements set forth by WCAG 2.0 (2008). The goal of Build for All is to provide various members of the startup community with explicit processes on how to successfully implement accessible options, we accept the fact that startups are already overburdened and are being pulled in many different directions. Build for All is not about making the case for inclusion, it is about providing tools to facilitate the education and implementation of inclusive products and services.

\section{Build for All Goals:}

The objectives of Build for All were as follows:

1: Create an online platform that contains information regarding the development of accessible startups and companies, including teams, products and environments.

2: Conduct and create content regarding the implementation of accessibility strategies in technology and web development in order to allow individuals with physical disabilities to access new services and products.

3: Provide the community with educational resources on how to successfully implement accessible options, while addressing the fact that startups are already overburdened and are being pulled in many different directions.

4: Foster an inclusive mindset and provide tools to facilitate the education and implementation process. 
Once Build for All was complete, the researcher then tested the willingness of startup team members to adopt accessible practices once provided with explicit actionable and concise resources.

\section{The Business Case:}

When accessibility is taken into consideration, individuals with disabilities will be able to more fully participate in the online world. Build for All looks to close the gap in the digital divide for those with disabilities. The startups benefit by learning how to adopt accessibility standards.

The current business case for increasing accessibility is that startups can increase their reach, and subsequently their profitability, if they are inclusive. Accessibility improves: SEO, usability, use by aging populations, reduced website costs (through reducing bugs and interoperability issues), and increased efficiency of the site through reduced bandwidth. If the platforms and services startups offer are difficult to use or maneuver, slow to load, or altogether inaccessible for those with physical, age induced, or developmental disabilities, purchases and use of the startup's solution will be reduced, or fail to grow due to lack of inclusivity.

If products and services are inaccessible, complaints and lawsuits can be filed against the startup on the grounds of discrimination or unequal access. In accordance with the AODA, maximum fines for a "person and unincorporated organizations that are guilty of a major offence under this Act can be fined up to $\$ 50,000$ dollars for each day the violation continues" (Cohen Share, 2011). As most startups are incorporated, this fine could be as high as $\$ 100,000$ per day that the violation continues (Cohen Share, 2011). Startups are traditionally low on resources and are fueled by their potential for growth. In the process of growing, startups may overlook necessary requirements in order to be the first to market, or lower the amount of time team members are working without a source of income. 
Build for All looks to be a tool used by startups to facilitate adoption of accessibility tactics to promote inclusivity, and lower the risk of violating web and access standards. Build for All differs from the pre-existing WAI, WCAG 2.0, W3C, AODA as it is focused on how to make startups accessible and inclusive through the use of actionable steps and examples (See Appendix D), whereas the aforementioned resources focus on why.

Build for All features job-specific perspectives tailored to the responsibilities of each individual within a startup, namely: developers, designers, marketers, and CEOs (See Appendix E). Each job has different necessities and requirements when it comes to making a startup accessible. Build for All provides concise and actionable resources that simplify the improvement of accessibility process as it pertains to specific roles within a startup. In this way, each role is able to take action in their role to make their products and services accessible to everyone.

\section{Literature Review}

In order for an idea to be considered an opportunity it must be durable, timely, attractive, and valuable (Wise \& Feld, 2017, p. 14). While startups may be working to secure investment and customers, they are forgetting about another significant demographic that has buying power, as well as the ability to recommend or berate products and services. Namely, end users with disabilities. In order to provide value to the masses, features must be included that dissolve the digital divide. The digital divide refers to "the gap between those who have and do not have access to computers and the Internet" (van Dijk, 2006, p. 221).

\section{The Digital Divide:}

The digital divide not only applies to those without access to the internet and technology, but also those who do not have ability to use technology through created barriers (Ragnedda \& 
Muschert, 2017, p. i). Unequal access is prevalent online and in social structures in regard to disability. According to Ragnedda and Muschert (2017), "theories of disability have gone ahead in leaps and bounds, yet such conceptual innovation has not been sufficiently registered in the framing of digital inequalities by both specialist and non-specialist researchers" (p. 67). In regard to human rights, digital technologies are becoming more involved in the battle for equality for those with disabilities (Ragnedda \& Muschert, 2017, p. 68). Stereotypes are prevalent in the description of disability, and many do not think of the term beyond the inability to see or hear, while in actuality there is a much larger scope of disabilities that are affected by improper use or lack or accessibility procedures.

Disabilities are created through the marginalization of individuals who have needs that differ from other individuals. According to the World Health Organization (2001):

Factors in a person's environment that, through their absence or presence, limit functioning and create disability. These include aspects such as: a physical environment that is not accessible, lack of relevant assistive technology (assistive, adaptive, and rehabilitative devices), negative attitudes of people towards disability, services, systems and policies that are either nonexistent or that hinder the involvement of all people with a health condition in all areas of life (p. 214)

From this, it could be concluded that the lack of inclusion in the world is the cause of disability, rather than the individual. If systems were in place to improve, not hinder, interactions on all levels, disabilities would be non-existent. Disabilities are a construct of a world ill-suited to heterogeneous differences.

Accessibility:

There are numerous principles related to designing accessible products and businesses. Don Norman (2013) in his work The Design of Everyday Things outlines the idea of universal design. When designing products and services, it is beneficial to consider every use-case for the product, business, or services being built. To illustrate the importance of universal design, Norman discusses an invention created by Sam Farber. Farber developed a specialty vegetable 
peeler for his mother who struggled with arthritis. Farber's peeler featured a large thick soft-grip plastic handle that increased the ease with which his mother could peel vegetables. The vegetable peeler's design factored in his mother's unique requirements, however, when introduced to the mass market, Farber approached marketing the peeler as a device that everyone could use (Norman, 2013, p. 244). Farber's vegetable peeler was such a success that he extended the design to other household products and began the business OXO (Norman, 2013, p. 244). Norman notes that successful products that are targeted or made to increase accessibility are not required to restrict themselves to individuals looking for accessible products, but rather, should focus on the practice of universal design for all.

Startups and Accessibility:

Startups should embrace inclusive and accessible tactics when developing their products and services, which, according to the low percentage of startup websites that are accessible, is not considered the standard today. This is not the usual practice as startups are already focusing on numerous tasks with limited resources in order to get their company off of the ground, and are looking for the path of least resistance (Paternoster, Giardino, Unterkalmsteiner, Gorschek, \& Abrahamsson, 2014, p. 1202). If information on standards and best practices is not clear, easy to navigate, easily adopted, or is seen as non-essential, startups look for an alternative source or forgo implementation altogether. The intent of this research is to increase willingness and adoption of accessibility practices within startups housed incubators in downtown Toronto. By testing the willingness of startups to include accessible practices once given explicit actionable and concise resources, this project looks facilitate and contribute to the knowledge of accessible practices in startups and aid in determining factors that lead to adoption. In addition, Build for All, as a web resource, should encourage startups to adopt practices which in turn create an inclusive atmosphere and increase their reach during their process of growth. 
While addressing accessibility and the requirements of the web to be inclusive, a gap still remains between the requirements and implementation. Unequal access to the Internet further marginalizes the population and subsequently affects business. There are numerous reasons in favour of making websites accessible, including increased profit and customer base, however startups are still not adopting accessible practices. There are resources that exist on why startups should be accessible, but a lack of resources that focus on how to implement accessibility.

\section{The Gap:}

Guidelines on accessibility exist, as do penalties for noncompliance, yet most websites remained inaccessible. Initial interviews illustrated anecdotally that the reason for noncompliance in startups is not a lack of why it is a lack of how. Startups thought about accessibility, but not regarding their products on the web, rather the accessibility of the space they occupied. They ensured that their space had elevator access or made sure that service animals were allowed, but over $80 \%$ of startup websites failed web accessibility standards (as shown in Appendix A). Given the right resources, which are both concise and actionable, startup founders would embrace inclusivity and develop with everyone in mind. If given the how, startups will adopt accessible practices. Due to the timeframe of this research there is not enough time to confirm adoption, but instead will track the increase in individuals' willingness to adopt.

\section{Methodology}

The goal of the research was to determine if startups are more likely to incorporate accessibility practices once the how is provided. The methods used involved pre-and postsurveys involving participants' understanding and willingness to implement accessibility protocol before and after being introduced to Build for All. The participants were first tested for their baseline willingness to incorporate accessibility practices through the initial survey. After 
which, the participants were given access to the Build for All web resource (www.buildforall.ca), and were given two weeks to review the resource. The two-week period was in place in order for the participant to have sufficient time to familiarize themselves with the platform. The secondary survey took place two weeks after the initial survey, and included a confirmation that the participant reviewed the platform prior to the post-survey. Any participant that did not review the platform was excluded from the study and subsequent results.

The inclusion criteria of the study were as follows: the individual must work at a startup, with between four (4) and fifteen (15) team members, in Toronto's downtown core within a design, communication, development, or management capacity. This includes CEOs, developers, communication officers, chief technology officers, graphic designers and marketing. Anyone who does not work at a startup, or who works at a startup with more than fifteen (15) team members, and less than four (4) team members, or those that work in sales or customer support are excluded from the study. Startup teams and members beyond of Toronto's downtown core were also excluded from the study. Sales and Customer Support have been excluded as they do not create, make, or design content that will be placed on the web. The focus of the study is on people who have the ability to take action to make startup online products more accessible.

Toronto's downtown core has a high density of startups in a small area. It is highly representative of a large number of startup types and features a lot of team diversity as opposed to other regions in Canada (The Corridor, n.d.). In short, the data collected in this geographic region is representative of this industry. All of the articles on Build for All have been directed at individuals that work in the development, marketing, or managerial capacities of a startup. All recruiting was conducted in person as it demonstrates a higher return on investment. This is due to entrepreneurs being focused on in-person networking, as social capital is perceived by CEOs 
as important to the success of the startup (Greve and Salaff, 2003). The likelihood of a response increases if the entrepreneurs and their team are interacting with a real person and increasing their social capital.

\section{Findings}

Firstly, it should be noted that the sample size for this research was small, which subsequently led to a small response rate and little observed impact. This in turn undermines the reliability of the findings. It is believed that the low rates were due to the amount of effort perceived by the startups in participating in the research. Over 140 individuals were approached for this research, of which 40 verbally agreed to participate during the window allocated to collecting data. 15 individuals then completed the pre-survey, and only 9 participants completed all study requirements. After conducting 9 pre- and post-surveys there was a $5 \%$ increase in the willingness of individuals to implement accessibility (Table 1.1). 7 participants believed that accessibility was very important to consider as a part of their business. Participants' understanding of accessibility guidelines increased by $25 \%$ after interacting with Build for All. In addition, there was a $7 \%$ decrease in the difficulty of following accessibility protocol, and a $26 \%$ increase in the amount of time spent implementing accessibility. All participants were willing to implement accessibility, however, a gap between willingness and implementation remained. The study demonstrated that participants were $14 \%$ more willing to encourage colleagues and team members to implement accessibility practices. Of the 9 participants, 9 were willing to implement accessibility, but only 1 participant was taking action to make sure that their products and services were accessible. 


\section{Table 1}

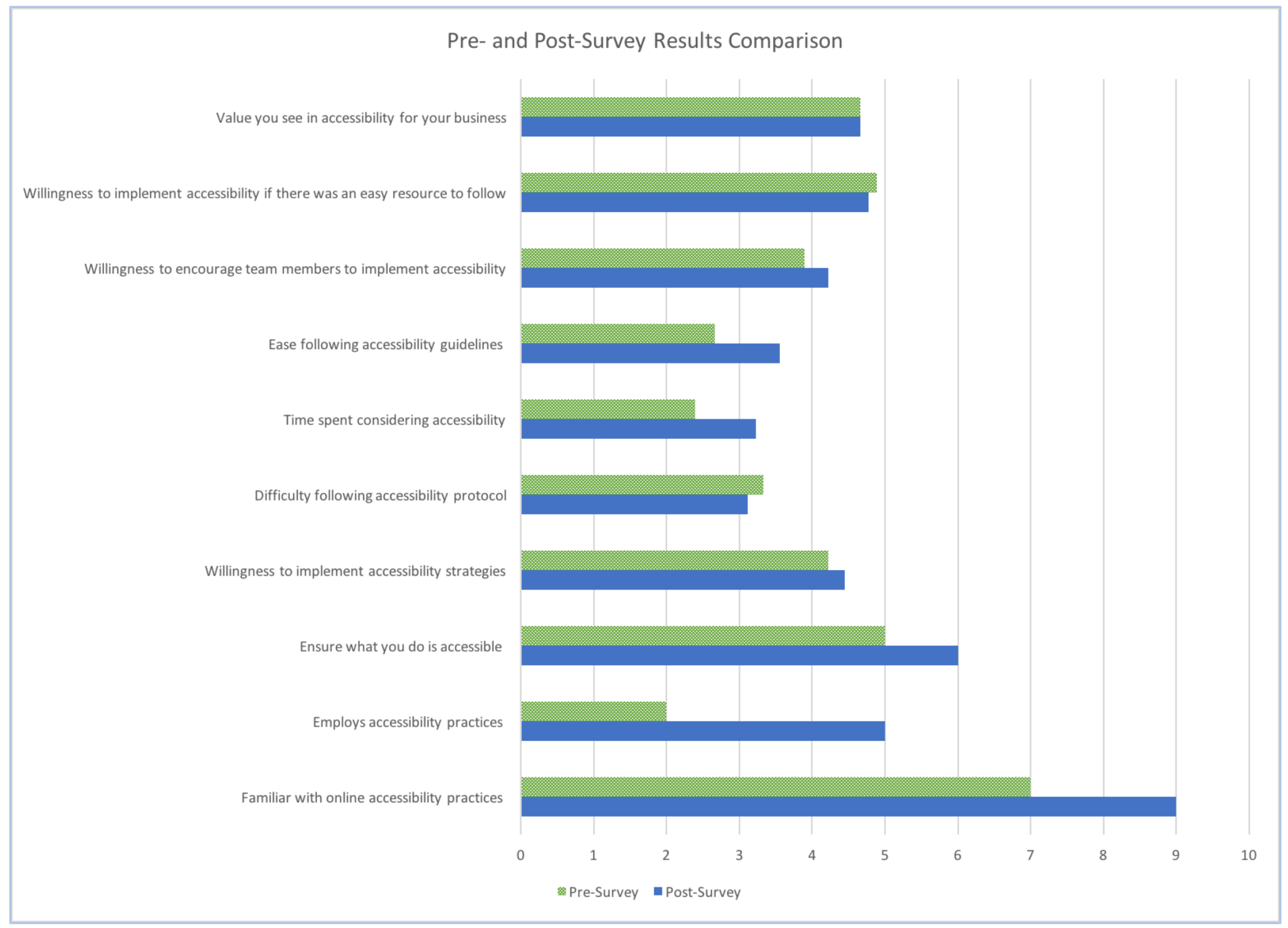

Table 1: The pre-and post-survey results are the average scores of the participants out of 5. As familiarity with online accessibility practices was a yes or no question, the results are based off the total number of participants that responded with yes.

8 of 9 surveyed post intervention found that Build for All increased their understanding and willingness to implement accessibility strategies. As demonstrated in Table 1.1, there was no change in the value perceived in accessibility for businesses, willingness to implement accessibility, and willingness to encourage team members to implement accessibility. This can be attributed to already high levels of willingness and perceptions of value found in making businesses accessible in the pre-survey. There was a strong increase in the ease following 
accessibility guidelines and time spent considering accessibility. While participants were willing to implement accessibility in the pre-survey there was difficulty understanding accessibility protocol and little time spent considering accessibility. The increase is most likely attributed to Build for All's resources, tools, and the provision of accessibility definitions. By reviewing Build for All, participants could definitively say that they spent time considering accessibility, and as such demonstrates a marked increase in results. Resources did not significantly make it less difficult to follow accessibility protocol. There was a decrease in the difficulty associated with following accessibility protocol, but the low impact can be attributed to participants becoming aware of more requirements than they were previously aware of. The amount of time spent on Build for All for each participant also varied, which can explain the low change rate. The biggest impact was in startups employing accessibility practices, individuals ensuring what they do is accessible, and familiarity with online accessibility practices. By spending time on Build for All, participants were able to see $\boldsymbol{h o w}$ to employ accessibility practices and in turn were able to ensure what they do was accessible by employing accessibility practices. The increase with familiarity in online accessibility practices can also be attributed to the definitions and resources provided on Build for All. By clarifying terms and acronyms, participants were able to better to comprehend protocol and practices. In order to conclusively determine if startups' willingness to adopt accessibility increases once given $\boldsymbol{h o w}$ to implement accessibility requirements requires additional time in order to approach more participants.

\section{Analysis}

When conducting the pre- and post-surveys there were numerous potential participants that appeared to be overwhelmed by the requirements of the study. As the study required the participants to fill out two surveys in the presence of the primary investigator over a period of 
two weeks, in addition to reviewing an online resource, many potential participants viewed this as too much effort for the purposes of research that would distract from their day to day operations. There was difficulty in finding participants in the condensed time period that the study required. This study would have benefitted from a longer research window in order to find more conclusive results and increase the number of participants in the study.

Individual perceptions of the definition of accessibility vary greatly (Persson, Åhman, Yngling, and Gulliksin, 2014). Those that participated in the research likely held their own views on what the definition of accessibility is. The willingness of all participants was rated very high in the pre-survey leaving little room for improvement. There were numerous other participants that only completed the pre-survey and as such were dropped from the research. The scope of this research decreased significantly due to the number of participants that did not complete the post-survey.

A potential cause for this may be due to entrepreneurship not having a thoroughly developed research practice, as mentioned by Hitt, Ireland, Camp, and Sexton (2001), stating, "although entrepreneurship has existed as a practice and field of study for quite some time, there is no commonly accepted and well-developed paradigm for research in the field" (p. 488). While only 9 participants completed both the pre- and post-surveys, for the purposes of user testing, one learns $85 \%$ of insights from the first five (5) participants, $98 \%$ after receiving feedback from ten (10) participants, and 100\% after fifteen (15) participants (Nielsen, 2000). Neilsen (2000) also recommends three (3) sessions of five (5) participants in order to approach the feedback of the first round of feedback iteratively. What I learned from my first 5 participants was that startups were already willing to make startups accessible, however the knowledge gap between why they should implement accessibility and how lowered, as a large difficulty that startups have 
is understanding accessibility protocol. In addition, due to the difficulty that was had with startup commitment, there may be a lack of commitment in accessibility due to the inherent characteristics of startup mentality, and that a longer time frame for additional iterations would be needed. For future research purposes, it is recommended that a focus group be the research method selected as it increases the likelihood of the participants to complete both surveys as most of the participant drop-off occurred in the two weeks given to review Build for All.

\section{Discussion}

The findings were greatly limited by the scope and timeline of the research. While not irrefutable due to the participant size, this study has found that: the majority of startups are willing to implement accessibility strategies only after being provided with Build for All, and even more willing to encourage colleagues to implement accessibility practices. It is simpler to encourage someone else to take responsibility, as there is less effort and assumed risk involved in passing on accountability than to take action for one's self, Shapiro (2017) describes this as "where the sense of ownership of what one says, does or thinks is surrendered or avoided by the individual, it is experienced by [them] to be elsewhere" (p.129). To lower the feeling of risk associated with task ownership "responsibility is assigned to some authoritative external figure or movement" (Shapiro, 2017, p.129). This also applies to startups and the implementation of accessibility. It is much easier to shift responsibility to another team member and be seen as "elsewhere" than to do the necessary work to make one's own work accessible.

Willingness of individuals to employ accessibility strategies were rated very high in the pre-survey leaving little room for improvement between the pre- and post-survey. If participants were willing to implement accessibility why haven't they done so already? This could be related to effort. It takes little to no effort to say that one is willing to take action, but it takes 
significantly more time, energy, and effort to execute. There is a difference between planning and doing. Kurniawan, Seymour, Talmi, Yoshida, Chater, and Dolan substantiate this, as "effort acts to discount the value of an action, an effect reflected in lower ratings and lower preference for options with high effort" (2010, p.318).

The decrease in difficulty involved in following accessibility protocol can be attributed to the difference between tacit and explicit knowledge. Tacit knowledge, as made famous by Dr. Ikujiro Nonaka, is "subjective and experience based knowledge that cannot be expressed in words, sentences, numbers or formulas, often because it is context specific", while explicit knowledge is "objective and rational knowledge that can be expressed in words, sentences, numbers or formulas" (Spencer, 2017, p. 1). Build for All focused on how to employ accessibility strategies, and as such made tacit information explicit. Humans have different methods of learning, both by understanding and doing. Socialization, or interaction between two or more individuals, is a requirement in sharing tacit knowledge (Spencer, 1997). Much like learning to ride a bike, individuals may understand how riding a bike works, may have both seen others riding bikes and have been told the basics of pedaling, but there is a gap between understanding and doing. One can only fully understand riding a bike after attempting to ride. This process of making tacit knowledge explicit is known as externalization (Spencer, 1997). By providing a multi-modal method of learning, by explaining why and providing how, with examples, Build for All makes tacit information explicit. Explicit knowledge is more easily shared and spread than tacit knowledge, and is suitable for knowledge transfers of a large scale through information technology (Spencer, 1997). 


\section{Conclusion}

The purpose of this research was to how the willingness of startups to adopt accessible practices would be affected, when startups were provided with how, instead of $\boldsymbol{w h} \boldsymbol{y}$. There are many online resources that focus on why websites should be accessible, both their reach and efficacy have been limited. There are numerous policies, standards, and laws enacted globally that require equal access for the web. Accessibility is a matter of equality and creating an inclusive environment so that individuals can interact online on their own terms. Despite the threat of fines and penalties for creating a non-accessible website, over $80 \%$ of websites do not meet accessibility standards.

Overall, based off the results of post-survey, the most significant increase was in the amount of time the individual perceived they spent considering accessibility and the individual's perceived capacity to understand online accessibility protocol. There is value in being shown how to carry out accessibility strategies, over why alone, as it conceptually lowers the burden of the individual. Being given explicit instructions simplifies the process for startups, and removes the uncertainty from the task. A prime example of this is a Lego set. The difficulty of the task increases based on the number of parts, and whether or not an instruction manual is present. One may understand why they want to build the set, but finds difficulty in completing the task if not given the instructions. Understanding how is crucial to successful execution, but knowing how is not always enough. Next steps in establishing the adoption of accessibility practices in startups involves revisiting the startups involved in the study in 6 months to determine whether accessibility practices were adopted by the startups on their websites.

The question then becomes: If given how, will startups increase their willingness to adopt accessible strategies? 
Based off the low participation rates of this study, further research on startup commitment and motivation to adopt new practices is required. Identifying the gap between motivation and implementation is the cornerstone of adoption. There is a need for greater focus on commitment to adopting new practices, in future research so as to better understand the timelines that would be required in order to follow-up with adoption in further studies.

In the end, all but one participant found that Build for All was a useful resource that positively impacted their ability to understand and implement accessibility practices. This paper proved there was an increase in understanding of accessibility standards and the amount of time spent considering accessibility. Future research should explore if providing how fosters adoption and closes the gap between willingness and implementation. 


\section{Appendix A}

Percentage of DMZ startup websites that passed and failed website accessibility standards. Error refers to websites that could not be scanned for accessibility purposes.

\section{Startup Website Accessibility Results}

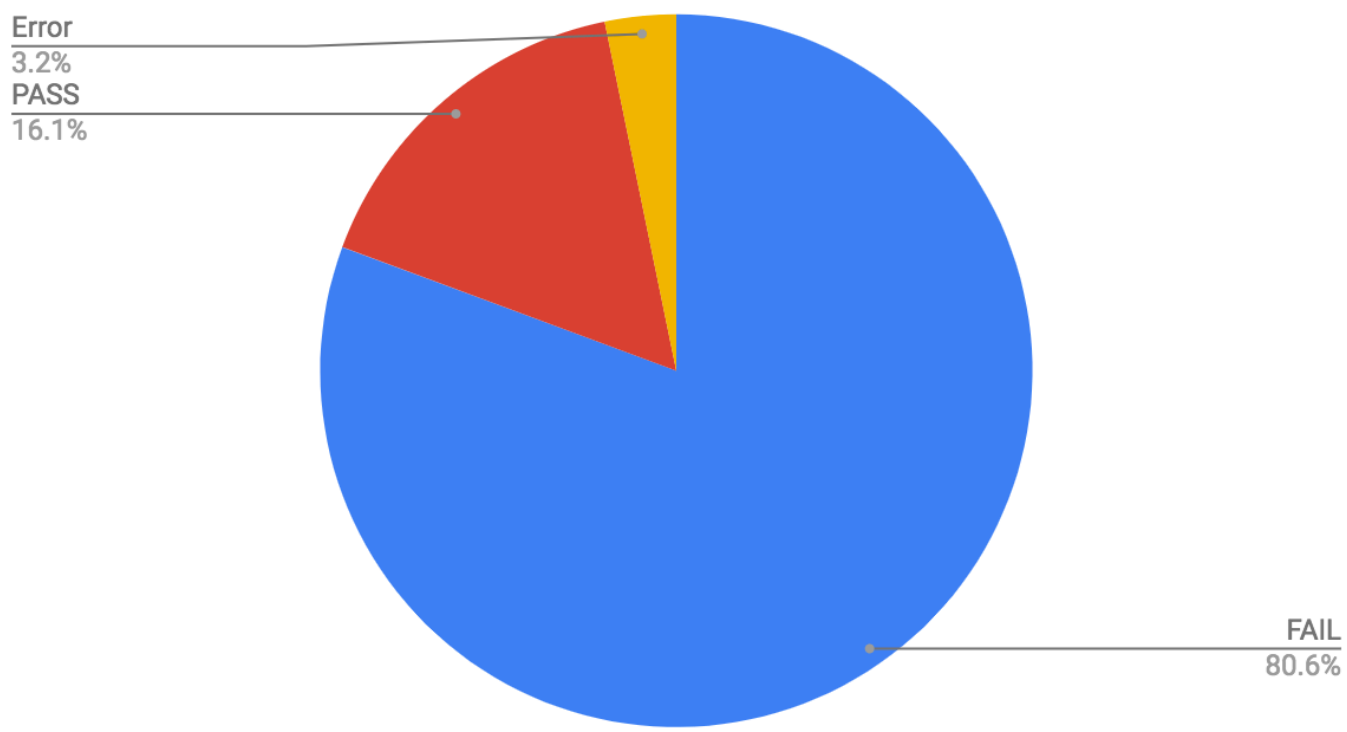


Appendix B

The average number of errors on DMZ startup websites that failed accessibility standards.

Number of Accessibility Errors

250

200

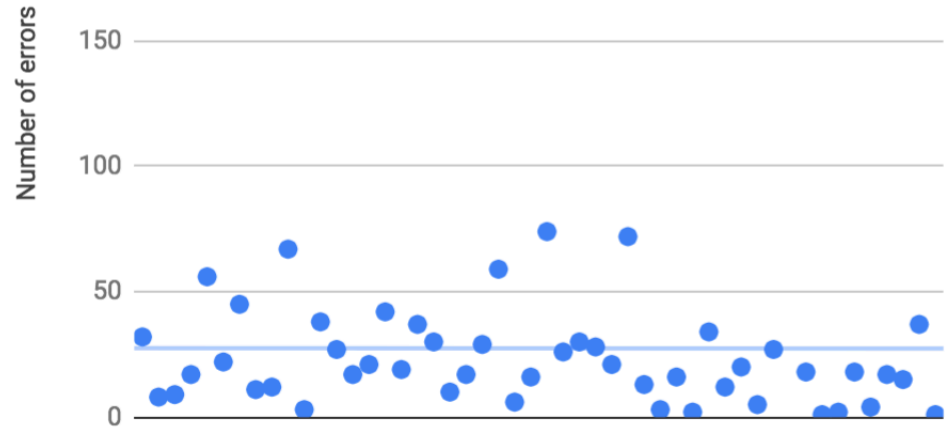

Startups with Failed WebAccessibility 


\section{Appendix C}

Build for All contains information regarding the development of accessible teams, products, and environments

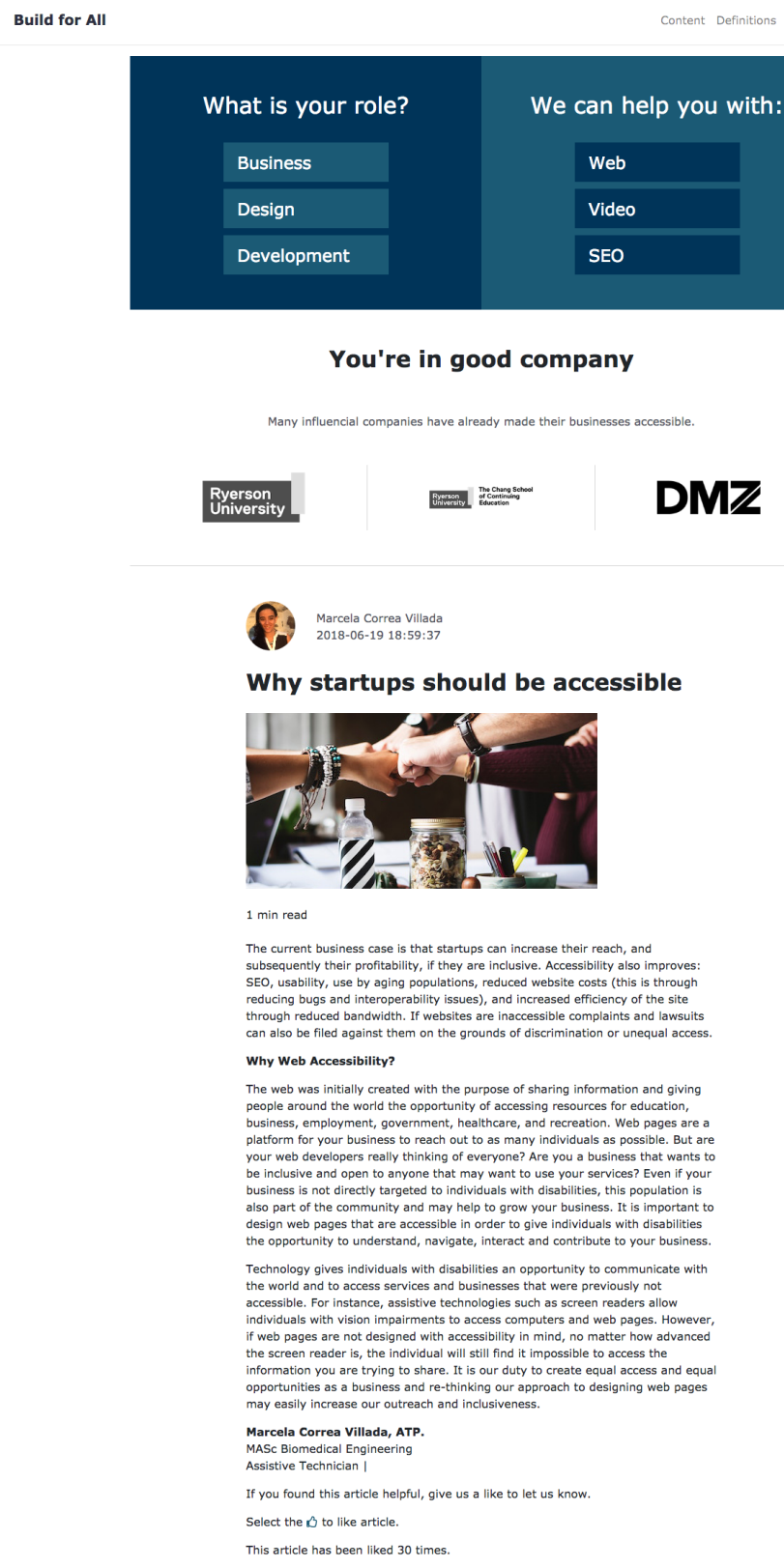

Startups for a better world

Let's chat.

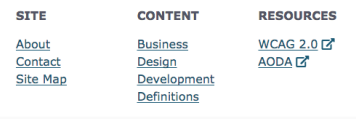

Privacy \& Terms $\quad$ Contact

() Build for All 2018 


\section{Appendix D}

Build for All provides explicit examples and how-to instructions

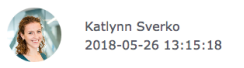

Keyboard Access and Navigation

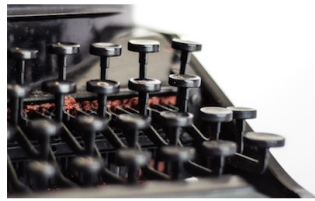

2 min read

Many people who have visual disabilities or require keyboard only access require
items on the page to be focussed and selectable without the click of a mouse or
finger ta.

finger tap. This is important to consider when creating buttons, text boxes, and

use touch sources like mice, touchpads, or tap with their fingers, use the tab key

on the keyboard to navigate interactive elements on a web page.

In order for you to make your website accessible to the tab key navigation you

The tabindex attribute moves sequentially based on the order of the HTML source.

Use tabinde $x=" \|$ "in order for the navigation to follow the natural order. For the

and act in a predictable manner, tabindex=" " $"$ " is the best way to implement this accessibility technique. Please see the Caution section below for tabindex values that are not equal to 0 .

It should be noted that some elements are focusable by default. Keyboard

interactions are automatically registered by the browser if using one of the

following HTML elements:

1. Anchor element including href attribute

2. Area element including href attribute

3. Button element

4. Input element

5. Select element

6. Text area element

The tabindex attribute should be used if repurposing or looking to add focus to an HTML element that is not focusable by default. If you are looking to make additional HTML elements focusable, such as anchor, area, container units like div atribute is the best way to do this.

Example:

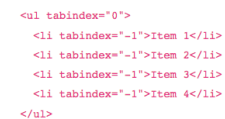

Another example:

<h1 tabindex=" "0" $>$ Tit 1e </h1>

$<\mathrm{p}$ tabindex-" $0 ">$ Some paragraph text $</ p$

<ing tabindex="0" sre="\#" alt="Ixarple inage

Caution:

If you begin using tabindex with numbers beyond 0 the navigation can get very messy and confuse the user. Only use tabindex with a number beyond 0 if there is an absolute necessity for aninterative element to be accessed first. th is net

usually a good idea.

If using a negative number the such as tabindex $=" 1$ " the user cannot navigate to the element, but the information it is associated with will be placed as a focusable element that will be addressed by the script.

If you found this article helpful, give us a like to let us know.

Select the $B \hat{b}$ to like article.

This article has been liked once.

Startups for a better world

$\begin{array}{lll}\text { SITE } & \text { CONTENT } & \text { RESOURCES } \\ \text { About } & \text { Business } & \text { WCAG 2.0 【' } \\ \text { Contact } & \text { Design } & \text { AODA } \\ \text { Site Map } & \text { Development } & \\ & \text { Definitions } & \\ & & \end{array}$


Appendix E

Job-specific categories with articles targeted at profession

Build for All

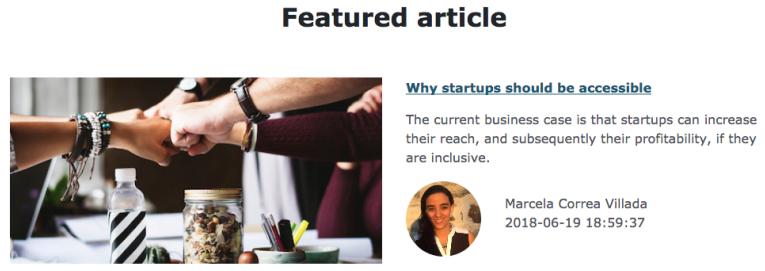

BUSINESS

DESIGN

DEVELOPMENT

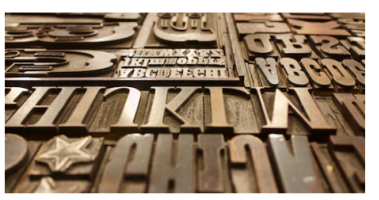

Alt-text for Developers

By providing alt-text for images you are allowing

someone to understand what is happening on a page,

whether or not they are able to see it.

(1)

Katlynn Sverko

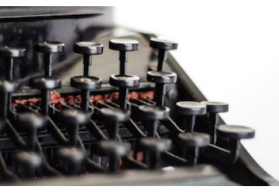

Kevboard Access and Navigation

Many people who have visual disabilities or require focussed and selectable without the click of a mouse or

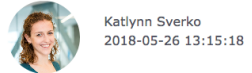

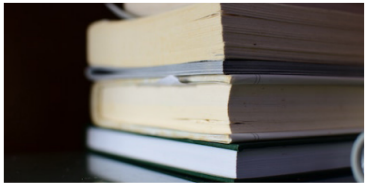

Skipping Repetitive Information and Long Lists

Providing alternative navigation that bypass long lists

(A) Katlynn Sverko $2018-05-26$ 13:33:43

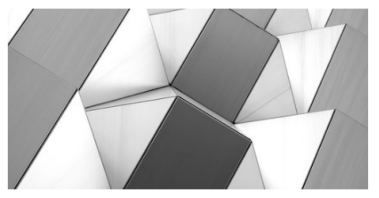

Accessible Text - Contrast and Text Visibility

The first step to accessibility in marketing is making

C.i. Katlynn Sverko $2018-05-26$ 13:46:00

Startups for a better world

Let's chat.
CONTACT US

\begin{tabular}{|c|c|c|}
\hline SITE & CONTENT & RESOURCES \\
\hline $\begin{array}{l}\frac{\text { About }}{\text { Contact }} \\
\text { Site Map }\end{array}$ & $\begin{array}{l}\text { Business } \\
\text { Design } \\
\text { Development } \\
\text { Definitions }\end{array}$ & $\frac{\text { WCAG } 2.0 \square^{\prime}}{\text { AODA } \square^{\prime}}$ \\
\hline
\end{tabular}




\section{References}

Accessibility for Ontarians with Disabilities Act 2014 (ON) (Canada). Retrieved April 24, 2018, from https://www.aoda.ca/the-act/

Cohen Share, S. (2011, August 25). AODA administrative Monetary Penalties Scheme - Three Strikes You're Out! Retrieved April 16, 2018, from https:/www.aoda.ca/aodaadministrative-monetary-penalties-scheme---three-strikes-you're-out/

Dardailler, D. (2009, June). WAI History. Retrieved April 23, 2018, from https://www.w3.org/WAI/history

Disability Rights Commission. (2004). The Web: Access and Inclusion for Disabled People; A Formal Investigation conducted by the Disability Rights Commission (Rep.). Retrieved April 23, 2018, from The Stationary Office website: https://www.city.ac.uk/ data/assets/pdf file/0004/72670/DRC Report.pdf

Greve, A., \& Salaff, J. W. (2003). Social networks and entrepreneurship. Entrepreneurship Theory and Practice, 28(1), 1-22. doi:10.1111/1540-8520.00029

Hitt, M. A., Ireland, R. D., Camp, S. M., \& Sexton, D. L. (2001). Guest editors' introduction to the special issue strategic entrepreneurship: Entrepreneurial strategies for wealth creation. Strategic Management Journal, 22(6/7), 479-491.

Information Technology and Innovation Foundation. (2017, March 8). 92 Percent of Most Popular Federal Government Websites Fail to Meet Basic Standards for Security, Speed, Mobile Friendliness, or Accessibility, New ITIF Study Finds. Retrieved April 24, 2018 , from https://itif.org/publications/2017/03/08/92-percent-most-popular-federal-governmentwebsites-fail-meet-basic

Kurniawan, I. T., Seymour, B., Talmi, D., Yoshida, W., Chater, N., \& Dolan, R. J. (2010). Choosing to make an effort: The role of striatum in signaling physical effort of a chosen action. Journal of Neurophysiology, 104(1), 313-321.

Miniwatts Marketing Group. (2018, June 2). Internet Usage Statistics: The Internet Big Picture World Internet Users and 2018 Population Stats. Retrieved July 22, 2018, from https://www.internetworldstats.com/stats.htm

Nielsen, J. (2000, March 19). Why You Only Need to Test with 5 Users. Retrieved July 8, 2018, from https://www.nngroup.com/articles/why-you-only-need-to-test-with-5-users/

Norman, D. (2013). The Design of Everyday Things. New York, NY: Basic Books.

Paternoster, N., Giardino, C., Unterkalmsteiner, M., Gorschek, T., Abrahamsson, P., Institutionen för programvaruteknik, . . . Blekinge Tekniska Högskola. (2014). Software 
development in startup companies: A systematic mapping study. Information and Software Technology, 56(10), 1200-1218. doi:10.1016/j.infsof.2014.04.014

Persson, H., Åhman, H., Yngling, A. A., Gulliksen, J., Medieteknik och interaktionsdesign, M., KTH, \& Skolan för datavetenskap och kommunikation (CSC). (2015). Universal design, inclusive design, accessible design, design for all: Different concepts - one goal? on the concept of accessibility - historical, methodological and philosophical aspects. Universal Access in the Information Society, 14(4), 505-526. doi:10.1007/s10209-014-0358-z

Pilling, D., Barrett, P., \& Floyd, M. (2004). Disabled People and the Internet. Joseph Rowntree Foundation. Retrieved July 22, 2018, from https://www.jrf.org.uk/sites/default/files/jrf/migrated/files/1859351867.pdf.

Ragnedda, M., \& Muschert, G. W. (2017). Theorizing digital divides. Routledge, an imprint of the Taylor \& Francis Group.

Shapiro, D. (2017). A psychodynamic view of action and responsibility: Clinical studies in subjective experience Routledge, an imprint of the Taylor \& Francis Group.

Spencer, B. (1997). Organizational Knowledge Creation: Ikujiro Nonaka. In Knowledge Advantage Conference. Retrieved July 22, 2018, from http://pagebaldwin.com/fa57/docs/KM_Organizational_Knowledge_Creation.pdf

The Corridor. (n.d.). Retrieved July 8, 2018, from https://thecorridor.ca/

United Nations Human Rights Council. (2011, May 16). Report of the Special Rapporteur on the promotion and protection of the right to freedom of opinion and expression, Frank La Rue, A/HRC/17/27. Retrieved April 23, 2018, from http://www2.ohchr.org/english/bodies/hrcouncil/docs/17session/A.HRC.17.27_en.pdf

van Dijk, J. A. G. M. (2006). Digital divide research, achievements and shortcomings. Poetics, 34(4), 221-235. doi:10.1016/j.poetic.2006.05.004

Vu, M. N., \& Ryan, S. (2018, January 02). 2017 Website Accessibility Lawsuit Recap: A Tough Year for Businesses. Retrieved April 24, 2018, from https://www.adatitleiii.com/2018/01/2017-website-accessibility-lawsuit-recap-a-toughyear-for-businesses/

WAI. (2016, May 6). Related Aspects of a Web for All. Retrieved April 24, 2018, from https://www.w3.org/WAI/intro/usable

Web Content Accessibility Guidelines (WCAG) 2.0. (2008, December 11). Retrieved April 12, 2018, from https://www.w3.org/TR/WCAG20/ 
Web Content Accessibility Guidelines (WCAG) 2.0. (2016). Understanding Conformance. Retrieved April 24, 2018, from https://www.w3.org/TR/UNDERSTANDINGWCAG20/conformance.html\#uc-levels-head

Wise, S., \& Feld, B. (2017). Startup Opportunities: Know When to Quit Your Day Job (2nd ed.). Hoboken, NJ: John Wiley \& Sons.

World Health Organization. (2001). International classification of functioning, disability and health. Geneva: WHO. p. 214 